\title{
Effect of dosage strategy on Al-humic flocs growth and re-growth
}

\author{
Wenzheng Yu, Chengzhi Hu, Huijuan Liu*, Jiuhui Qu \\ State Key Laboratory of Environmental Aquatic Chemistry, Research Center for Eco-Environmental Sciences, Chinese Academy of Sciences, Beijing 100085, China
}

\section{A R T I C L E I N F O}

\section{Article history:}

Received 1 November 2011

Received in revised form 11 March 2012

Accepted 25 April 2012

Available online 4 May 2012

\section{Keywords:}

Flocs breakage

Re-growth

One time dosage

Continuous dosage

Surface activity

\begin{abstract}
A B S T R A C T
The ability of flocs growth has a significant effect on the efficiency of solid/liquid separation during coagulation process. The growth and re-growth of flocs by one time or continuous dosage were explored at neutral $\mathrm{pH}$, including one time and continuous additional dosage strategy after flocs breakage. The size of flocs formed by continuous dosage before breakage was much larger than the one formed by one time dosage. There was significant irreversibility of floc breakage when no additional coagulant was added. For the one with one time initial dosage strategy, the size of re-grown flocs by one time additional dosage was nearly the same as that before breakage. The re-grown flocs formed by continuous additional dosage were a little smaller than that formed by one time additional dosage. It definitely showed that the growth or connection of flocs was probably not determined by the flocs size distribution and fractal dimension, but by the surface activity of flocs. Additional dosage could improve flocs re-grown ability because the surface activity of broken flocs was repaired by adding new alum.
\end{abstract}

(C) 2012 Elsevier B.V. All rights reserved.

\section{Introduction}

In water treatment, coagulation is an essential process for the removal of particulate and/or natural organic matter (NOM). Aluminum salt is commonly used as the flocculant to coagulate small particles into larger flocs that can be effectively removed in the subsequent separation process of sedimentation and/or filtration. The properties of flocs, such as size, structure and strength, have a significant effect on the efficiency of solid/liquid separation process [1]. During flocculation, the floc structure changes continually because the flocs internal bonds break under shear and re-form at more favorable points where the attractive force is greater or the repulsive force is smaller. When the balance between the rate of aggregation and the rate of breakage for a given shear condition is reached, flocs formation process completes and the size of flocs reaches a steady plateau [2]. Therefore, flocs breakage and recovery should also be taken into consideration for overall process optimization and have been the subject of in-depth research in recent years $[3,4]$.

Some works $[5,6]$ on flocs formed with NOM showed that it was difficult for the broken NOM flocs return to their size before breakage. NOM flocs re-growth after exposure to high shear was found to be limited for waters coagulated with alum, ferric sulfate and polyDADMAC [4]. Yukselen and Gregory [7,8] also found that only limited re-growth of kaolin flocs occurred indicated a significant irreversibility of the floc break-up process. Li et al. [9] also showed that broken kaolin flocs were difficult to re-grow when alum was

\footnotetext{
* Corresponding author. Tel.: +86 10 62849128; fax: +861062849160.

E-mail address: hjliu@rcees.ac.cn (H. Liu).
}

used. Normally, sweep coagulation is the dominant mechanism at optimal dosage of alum, and it is difficult for broken flocs to fully regrow to their initial size before breakage. Our previous research [10] indicated that there was significant irreversibility of floc breakage when no additional coagulant was added. When a small additional dosage of alum was added to the suspension during floc breakage, the size of re-grown flocs was a little higher than that before breakage according the floc monitoring results of flocculation index. The average size of flocs formed in two-stage addition mode roughly reached one half larger than that in one-stage addition mode [11]. Therefore, the freshly formed precipitate can improve the size of flocs, and continuous precipitate of coagulant by continuous dosage may change the growth and re-growth of flocs.

This work aimed to evaluate the grown and re-grown ability of flocs with different initial and additional coagulant dosage strategy to explore the flos formation mechanism using alum. Floc characteristics, including size and fractal dimension before and after breakage were investigated. Comparing with chemical coagulation, continuous electrocoagulation had higher removal of red dye in wastewater [12]. Therefore, comparison has been done between one time and continuous dosage on the performance of flocs growth and re-growth. An improved understanding of this process should be an important addition to knowledge in the field of NOM coagulation and removal.

\section{Methods and materials}

\subsection{Stock suspension and solution}

Five grams of humic acid, sodium salt (HA, Aldrich, Cat: H1, 675-2), was dissolved in deionized (DI) water, with $\mathrm{pH}$ adjusted 
to 7 , and mixed by a magnetic stirrer for $24 \mathrm{~h}$. The solution was diluted to $500 \mathrm{~mL}$ in a measuring flask and was stored in dark. Alum $\left(\mathrm{Al}_{2}\left(\mathrm{SO}_{4}\right)_{3} \cdot 18 \mathrm{H}_{2} \mathrm{O}\right.$, analytical reagent) was used as coagulant. Stock alum solutions were prepared at a concentration of $0.1 \mathrm{M}$ in DI water $(0.2 \mathrm{M} \mathrm{Al})$.

\subsection{Jar test}

For the flocculation tests, the stock humic acid was diluted in DI water $(1000 \mathrm{~mL})$, with $5 \mathrm{mM} \mathrm{NaHCO}$, giving test solution with a HA concentration of $10 \mathrm{mg} / \mathrm{L}$. During the jar test, the $\mathrm{pH}$ of final solution was maintained at 7.0 by prior addition of a predetermined amount of $0.1 \mathrm{M} \mathrm{HCl}$ or $\mathrm{NaOH}$. Initial dosage of $0.2 \mathrm{mM}$ was used here since the zeta potential was near $0 \mathrm{mV}$ [10]. The temperature was maintained at $25 \pm 1{ }^{\circ} \mathrm{C}$.

The model water was stirred at $50 \mathrm{rpm}$ for $60 \mathrm{~s}$ and then a certain dosage of alum $(0.2 \mathrm{mM}$ for one time or continuous dosage for $20 \mathrm{~min}$ ) was added, with a simultaneous increase of stirring speed to $200 \mathrm{rpm}$. The equipment used was a Flocculator ZR4-2 (Shenzhen Zhongrun, China), which enables mixing speeds and times to be preset. For initial one time strategy, the rapid mix speed of $200 \mathrm{rpm}$ was maintained for $1 \mathrm{~min}$, and then reduced to $50 \mathrm{rpm}$ for 15 min to allow floc growth to occur. Whereafter, the speed was then increased to $200 \mathrm{rpm}$ for $1 \mathrm{~min}$ to break the flocs, and then back to $50 \mathrm{rpm}$ for $10 \mathrm{~min}$ for the flocs to re-grow. At the end of broken stage, none or additional alum $(0.03 \mathrm{mM})$ was added by one time or continuous strategy $(0.01 \mathrm{mM} / \mathrm{min})$.

\subsection{Floc structural analysis}

A laser diffraction instrument (Malvern Mastersizer 2000, Malvern, UK) was used to measure dynamic floc size as the coagulation and flocculation process proceeded, which was used by many researchers $[4,13]$. The suspension was monitored by drawing water through the optical unit of the Mastersizer and back into the jar by a peristaltic pump on the return tube with $5 \mathrm{~mm}$ internal diameter peristaltic pump tubing. The inflow and outflow tubes were positioned opposite one another at a depth just above the paddle in the holding ports. Size measurements were taken every half a minute for the duration of the jar test and logged onto a PC.

Mass fractals may be summarized by the relationship between their mass $M$, a characteristic measure of size $L$, and the mass fractal dimension $D_{\mathrm{f}}$ :

$M \propto L^{D_{\mathrm{f}}}$

During small-angle light scattering, a light beam is passed through a sample. The particles in the sample scatter light proportionally to their size and at a constant angle independent of which part of the particle is hit by the beam. Small particles scatter light at high angles, while large particles scatter at lower angles. The Malvern Mastersizer has an array of photosensitive detectors at different angles between $0.01^{\circ}$ and $40.6^{\circ}$ that detect the light scattered by the sample. The determination of fractal dimension by use of SALLS has been well illustrated [14-16], so only a brief overview is given below.

The total scattered light intensity $I$ is a function of the scattering vector $Q$, where $Q$ is the difference between the incident and scattered wave vectors of the radiation beam in the medium [14], which is given by Eq. (2).

$Q=\frac{4 \pi n \sin (\delta / 2)}{\lambda}$

where $n$ is the refractive index of the suspending medium, $\delta$ is the scattering angle, and $\lambda$ is the wavelength of the radiation in a
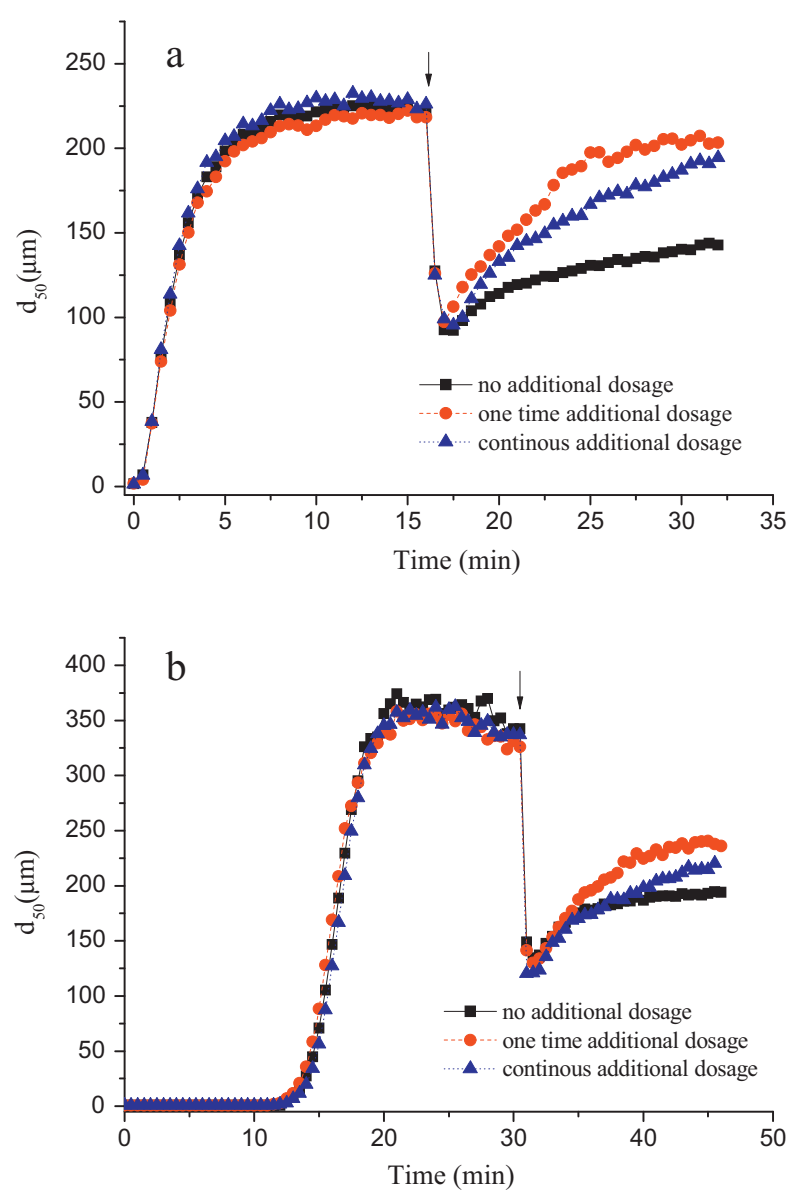

Fig. 1. Flocs formation, breakage and re-growth with different initial and additional dosage strategy: (a) one time initial dosage and (b) continuous initial dosage. Arrows show the beginning of a $60 \mathrm{~s}$ period of stirring at $200 \mathrm{rpm}$. Coagulant addition was at the end of a $60 \mathrm{~s}$ period of rapid mixing at $200 \mathrm{rpm}$ and followed by slow stirring at $50 \mathrm{rpm}$.

vacuum. For independently scattering aggregates, $I$ is the related to $Q$ and the fractal dimension by $D_{\mathrm{f}}(3)$ :

$I \propto Q^{D_{\mathrm{f}}}$

A confirmation of the power relationship by Spicer et al. [13] is to plot $I$ against $Q$ on a log-log scale. A power law relationship exists if this yields a straight line, the slope of which is used to give $D_{\mathrm{f}}$. The relationship only holds when the length of investigation is much larger than the primary particles and much smaller than the floc aggregates.

\section{Results}

\subsection{Grown and re-grown ability of flocs by different dosage strategy}

The variation of floc size $\left(d_{50}\right)$ with time during formation, breakage and re-growth of flocs coagulated with one time and continuous dosage strategy is shown in Fig. 1 . These all show the $d_{50}$ reaching a certain limiting or plateau value after a certain time by the initial dosage phase. This represents the steady state of flocs size, which depends on the floc strength and the effective shear rate in the stirred vessel. A rapid rise of $d_{50}$ occurs soon after the end of the rapid mix phase, and the $d_{50}$ reaches the plateau value after a few minutes. When the stirring speed is increased again, there is a rapid reduction in $d_{50}$, because of flocs breakage, and lowest recovery when the slow stirring speed is restored if there is 
no additional dosage of alum. Additional dosage of alum can significantly improve the re-grown ability of broken flocs whether by one time dosage or continuous dosage. From Fig. 1a, the improvement by one time additional dosage was higher than that by continuous additional dosage, and the size of re-grown flocs was nearly the same that before breakage.

While for continuous dosage of alum in the initial phase, there was long lag phase as there was no flocs due to low negative charge. During the lag phase, the aggregates were not yet large enough to give a measurable increase in $d_{50}$ value. Noted that the plateau $d_{50}$ value for continuous coagulant dosage strategy in Fig. 1b was significantly higher than that in Fig. 1a, where the total dosage of alum was the same and the zeta potential of flocs was also the same before flocs breakage. Also additional dosage of alum could significantly improve the re-grown ability of broken flocs whether by one time additional dosage or continuous additional dosage, although the size of re-grown flocs was smaller than that before breakage. Also the re-grown ability of broken flocs improved by one time additional dosage was higher than that by continuous additional dosage.

The different dosage strategy before and after breakage caused different grown and re-grown ability of flocs might be resulted from different surface characteristic of flocs. Before breakage, the surface of flocs was more active and continuous initial dosage of alum improved the surface activity better, while after breakage the surface of broken flocs was inactive, and continuous additional dosage only a little improved the activity of broken flocs. In contrast, one time additional dosage after breakage dramatically improved the surface characteristic of most of broken flocs, and then these flocs are reactive and can aggregate with other flocs, leading to higher re-grown ability.

\subsection{Effect of additional dosage strategy on flocs size distribution (FSD)}

Analysis of FSD from Fig. 1a showed a left shift of the major peak after breakage, undergoing more than half the original value (Fig. 2). There was also a considerable increase in the smaller peak at floc sizes between 10 and $40 \mu \mathrm{m}$. After the re-growth phase, this smaller peak reduced with a commensurate shift to the major peak. As shown in Fig. 2a, if there was no additional dosage of alum, the broken flocs were still smaller than that before breakage.

If there was one time additional dosage of alum added at the end of breakage phase, flocs FSD was much different. As shown in Fig. 2b, the frequency of flocs after breakage and one time additional dosage of alum, larger than $200 \mu \mathrm{m}$, were significantly higher than that after breakage without additional dosage of alum (Fig. 2a). After re-growth for $15 \mathrm{~min}$, the FSD data showed that the average size of re-grown flocs was nearly the same as the flocs before breakage. For continuous additional dosage of alum after breakage, floc FSD was found a little different from the one of no additional dosage. The frequency of flocs that was larger than $200 \mu \mathrm{m}$ by continuous additional dosage of alum was a little higher than that of flocs without additional dosage. Also after re-growth phase, the peak of FSD shifted to the right with bigger size of flocs comparing with the one without additional dosage, though the FSD was a little smaller than the one before breakage.

\subsection{FSD with same $d_{50}$ value before breakage and after re-growth}

In order to investigate the coagulation process in more detail, the size distribution of flocs before and after breakage with same $d_{50}$ value but different additional dosage strategy were investigated. Flocs samples of before breakage and after re-growth with different additional dosage modes (none, one time, continuous) were taken at the point where the size of flocs had reached $200 \mu \mathrm{m}$. The


Fig. 2. Effect of additional dosage strategy on flocs size distribution, conditions for Fig. 1a: (a) no additional dosage; (b) one time additional dosage; and (c) continuous additional dosage.

size distributions of flocs with the same $d_{50}$ value before breakage and those after re-growth are shown in Fig. 3. The FSD at an equivalent stage $\left(d_{50}\right)$ in flocs growth showed almost no difference between before breakage and after re-growth for the three additional dosage modes. However, it was clear from Fig. 1b that the flocs sizes before breakage was larger than that after re-growth 

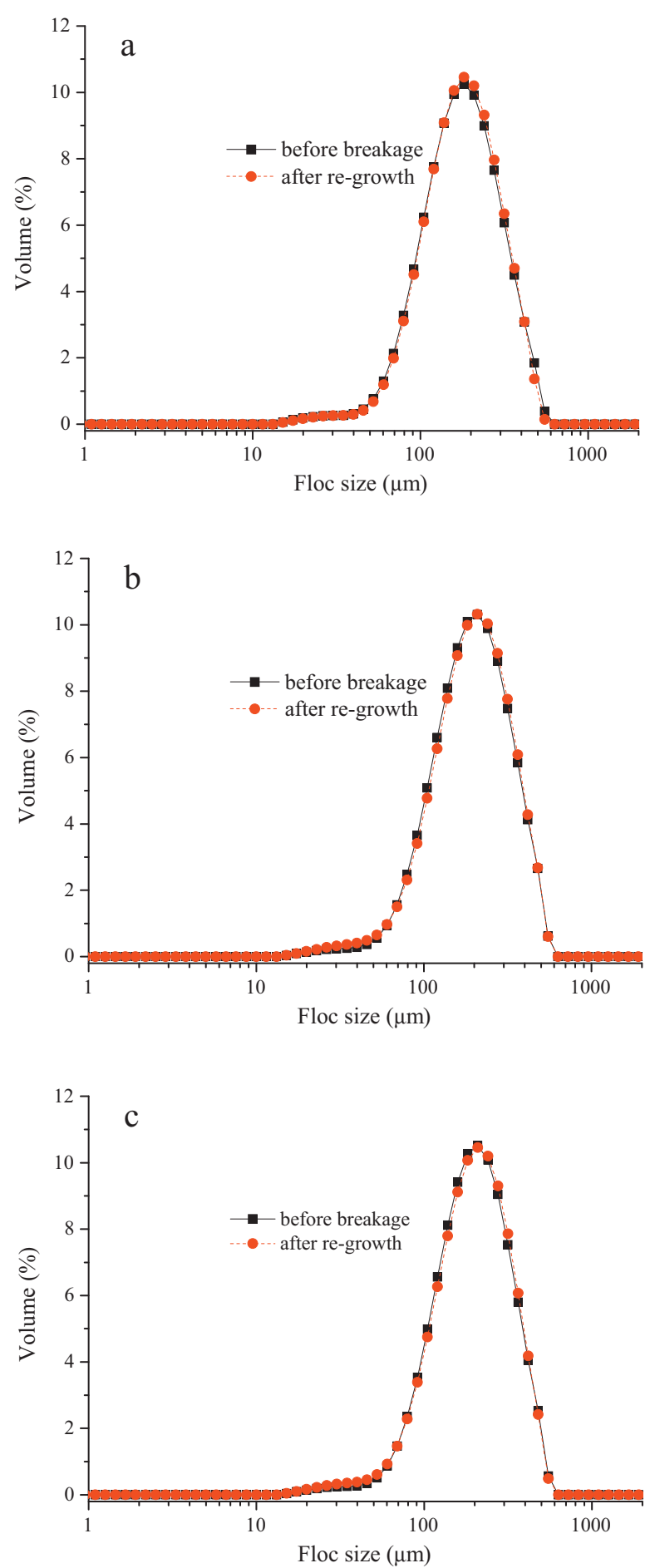

Fig. 3. Effect of additional dosage strategy on flocs size distribution with same $d_{50}$ value $(200 \mu \mathrm{m})$, conditions for Fig. $1 \mathrm{~b}$ : (a) no additional dosage; (b) one time additional dosage; and (c) continuous additional dosage.

regardless additional dosage strategy used. The results indicated FSD could not be changed by different additional dosage strategy. The reason for the different plateau $d_{50}$ values before breakage and after re-growth should be the different surface activity of flocs, in dependent of the FSD.

Amirtharajah and Mills [17] suggested a fast destabilization process (less than $1 \mathrm{~s}$ ) after alum dosage, so it was unlikely that the slow flocculation process would give any change in the degree of destabilization. Close examination of Fig. 1 showed that, for long

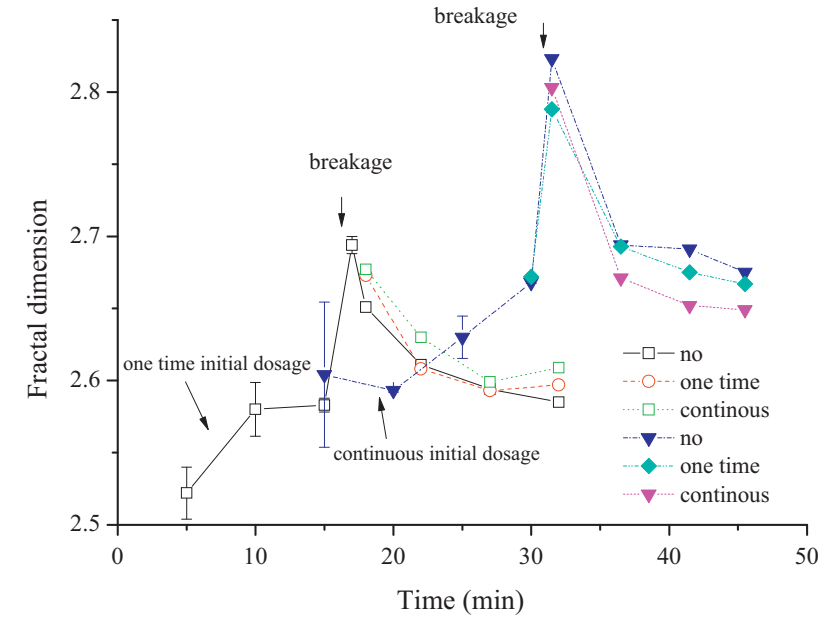

Fig. 4. Change of fractal dimension during flocs formation, breakage, and re-growth with different additional dosage strategy.

time continuous dosage of alum, the $d_{50}$ value approached high limiting value, which was significantly higher than that with one time dosage ( $50 \%$ higher). A limiting flocs size by one time dosage compared with continuous dosage implied that some flocs breakage in coagulation/flocculation process led to low plateau flocs size. In contrast, the surface of breakage flocs could be persistently activated by continuous dosage.

\subsection{Fractal dimension of flocs}

Curves were plotted for the coagulating suspensions as they were growing, broken, and subsequently re-growing. This demonstrated how the degree of flocs compaction changed with different shearing patterns for the small flocs. There was a considerable difference in NOM flocs fractal dimension for the different dosage strategy of coagulants (Fig. 4). Prior to breakage, one time initial dosage of flocs had the lower $D_{\mathrm{f}}$ though it increased from 2.52 to 2.60 when coagulated from $5 \mathrm{~min}$ to $15 \mathrm{~min}$, while flocs by continuous initial dosage appeared to have a higher degree of compaction with a $D_{\mathrm{f}}$ increased from 2.60 to 2.67. On exposure to high shear, the $D_{\mathrm{f}}$ value was seen to increase dramatically for both coagulant dosage strategy.

Once the original shear had been returned, flocs compaction was seen to decrease for both of the flocs with one time or continuous initial dosage. The additional dosage of alum could not improve the fractal dimension of re-grown flocs, and there was no significant difference between the one time and continuous additional dosage. So the fractal dimension of flocs should not be the factor influencing the grown and re-grown ability of flocs.

\section{Discussion}

The size of flocs is of significance for the flocs separation and purification, so the connection of flocs between micro-flocs should be researched. As researched by our previous paper [10], the additional dosage of alum can improve the size of flocs whether the flocs is broken or not. Also the result was supported in this paper. Continuous initial dosage of alum before breakage could significantly improve the size of flocs, which was half larger than the one formed with one time initial dosage. As shown in Fig. 4, the fractal dimension of flocs formed by continuous initial dosage was higher than that formed by one time initial dosage, while the size of flocs was larger by continuous dosage. Therefore, the fractal dimension may be not the factor influence the growth of flocs. From Fig. 3, the FSD of flocs before and after breakage with same 




Fig. 5. A schematic diagram of the variation of flocs activity in flocs formation, breakage process.

$d_{50}$ was nearly the same, while the grown and re-grown ability were significantly different. Surface activity was brought forward in our previous papers $[10,18]$, and here we put forward "active point". A schematic diagram is shown to explain the variation of flocs activity in flocs formation, breakage process (Fig. 5). Small active particle is formed after alum is added into the humic acid synthetic water. If added by one time dosage, less active cluster is formed as the cluster would also break in aggregation process, and the size of flocs reaches a plateau value after a few minutes. While if added by continuous dosage, formed cluster kept active as continuous active particles cover the cluster. Theses clusters with more active points are more prone to form larger flocs. Therefore, the number of active points determines the grown ability of flocs, which is less by one time dosage than that by continuous dosage. Thus, the size of flocs formed by continuous dosage of alum is larger than that of flocs formed by one time dosage.

Kobayashi et al. [19] considered that, the number of cluster-cluster bond(s) and the magnitude of cohesive force dominated the breakage of a floc. The large flocs will then be broken at the points corresponding to cluster-cluster bonds, depending on the relative magnitude of the cohesive force and the applied shear force. After flocs breakage, there was little active point on the surface of broken flocs, and re-grown ability of broken flocs was low if no additional dosage of alum was added. Additional dosage of alum at the point after flocs breakage improves re-grown ability of broken flocs because the inactive point is repaired by the new precipitate and the repaired flocs became more active to flocculate.

\section{Conclusions}

1. Continuous dosage of alum caused larger size of flocs comparing with one time dosage. However, the re-grown flocs formed by continuous additional dosage were a little smaller than that formed by one time additional dosage.

2. The size distribution of flocs before breakage and after re-growth in same $d_{50}$ value was nearly the same. So it was definite that the size distribution of flocs was not crucial for the growth or connection of flocs.

3. The fractal dimension of flocs formed by continuous dosage of alum was larger than that by one time dosage, while it may not influence the grown and re-grown ability of flocs.
4. The re-grown ability of broken flocs was improved by one time or continuous additional dosage because the surface activity of broken flocs was repaired by adding new alum.

\section{Acknowledgments}

This work was supported by National Natural Science Foundation of China (Grants 51108444 and 51138008). The authors are also grateful for financial support from the Creative Research Groups of China (No. 50921064) and the National High-Tech Research and Development Program (863) of China (No. 2009AA06Z303).

\section{References}

[1] W.Z. Yu, G.B. Li, Y.P. Xu, X. Yang, Breakage and re-growth of flocs formed by alum and PACl, Powder Technol. 189 (2009) 439-443.

[2] C.A. Biggs, P.A. Lant, Activated sludge flocculation: on-line determination of floc size and the effect of shear, Water Res. 34 (2000) 2542-2550.

[3] K. McCurdy, K. Carlson, D. Gregory, Flocs morphology and cyclic shearing recovery: comparison of alum and polyaluminum chloride coagulants, Water Res. 38 (2004) 486-494.

[4] P. Jarvis, B. Jefferson, S.A. Parson, Re-growth, and fractal nature of natural organic matter flocs breakage, Environ. Sci. Technol. 39 (2005) 2307-2314.

[5] W.Y. Xu, B.Y. Gao, Q.Y. Yue, Y. Wang, Effect of shear force and solution pH on flocs breakage and re-growth formed by nano- $\mathrm{Al}_{13}$ polymer, Water Res. 44 (2010) 1893-1899.

[6] Y. Wang, B.Y. Gao, X.M. Xu, W.Y. Xu, G.Y. Xu, Characterization of floc size, strength and structure in various aluminum coagulants treatment, J. Colloid Interface Sci. 332 (2009) 354-359.

[7] M.A. Yukselen, J. Gregory, The effect of rapid mixing on the break-up and reformation of flocs, J. Chem. Technol. Biotechnol. 79 (2004) 782-788.

[8] M.A. Yukselen, J. Gregory, The reversibility of flocs breakage, Int. J. Miner. Process. 73 (2004) 251-259.

[9] T. Li, Z. Zhu, D.S. Wang, C.H. Yao, H.X. Tang, The strength and fractal dimension characteristics of alum-kaolin flocs, Int. J. Miner. Process. 82 (2007) 23-29.

[10] W.Z. Yu, J. Gregory, L. Campos, Breakage, Regrowth of Al-humic flocs - effect of additional coagulant dosage, Environ. Sci. Technol. 44 (2010) 6371-6376.

[11] T. Liu, Z.L. Chen, W.Z. Yu, J.M. Shen, J. Gregory, Effect of two-stage coagulant addition on coagulation-ultrafiltration process for treatment of humic-rich water, Water Res. 45 (2011) 4260-4268.

[12] B. Merzouk, B. Gourich, K. Madani, C. Vial, A. Sekki, Removal of a disperse red dye from synthetic wastewater by chemical coagulation and continuous electrocoagulation: a comparative study, Desalination 272 (2011) 246-253.

[13] P.T. Spicer, S.E. Pratsinis, J. Raper, R. Amal, G. Bushell, G. Meesters, Effect of shear schedule on particle size, density, and structure during flocculation in stirred tanks, Powder Technol. 97 (1998) 26-34.

[14] P. Tang, J. Greenwood, J.A. Raper, A model to describe the settling behavior of fractal aggregates, J. Colloid Interface Sci. 247 (2002) 210-219.

[15] T.D. Waite, J.K. Cleaver, J.K. Beattie, Aggregation kinetics and fractal structure of Y-alumina assemblages, J. Colloid Interface Sci. 241 (2001) 333-339. 
[16] R.M. Wu, D.J. Lee, T.D. Waite, J. Guan, Multilevel structure of sludge flocs, J. Colloid Interface Sci. 252 (2002) 383-392.

[17] A. Amirtharajah, K.M. Mills, Rapid-mix design for mechanisms of alum coagulation, J. Am. Water Works Assoc. 74 (1982) 210-216.
[18] W.Z. Yu, J. Gregory, L. Campos, The role of mixing conditions on floc growth, breakage and re-growth, Chem. Eng. J. 171 (2011) 425-430.

[19] M. Kobayashi, Y. Adachi, S. Ooi, Breakup of fractal flocs in a turbulent flow, Langmuir 15 (1999) 4351-4356. 